\title{
VLBI Constraints on Type I b/c Supernovae
}

\author{
Michael F. Bietenholz ${ }^{1,2,3}$ \\ ${ }^{1}$ Hartebeesthoek Radio Astronomy Observatory, P.O. Box 443, Krugersdorp 1740, South Africa \\ ${ }^{2}$ Department of Physics and Astronomy, York University, Toronto, Ontario M3J 1P3, Canada \\ ${ }^{3}$ Email: michael@hartrao.ac.za
}

(Received September 16, 2013; ACCEPTED October 9, 2013)

\begin{abstract}
Very long baseline interferometry observations of supernovae and gamma-ray bursts provide almost the only way of obtaining spatially resolved information about the sources. In particular, a determination of the expansion velocity of the forward shock, as well as the geometry of the fireball and its evolution with time are possible for relatively nearby events, provided they are radio bright. Monitoring the expansion of the shock front can provide information on the density profiles of both the circumstellar material and on the ejecta. Very long baseline interferometry observations can also potentially resolve gamma-ray burst jets which are not directed along the line of sight, providing crucial confirmation of relativistic expansion in such objects. This review gives an overview of recent results from supernovae, including the Type I b/c SNe $2011 \mathrm{dh}, 2009 \mathrm{bb}$, and $2007 \mathrm{gr}$, and discusses the prospects for future observations.
\end{abstract}

Keywords: supernovae — radio continuum

\section{INTRODUCTION}

Type I b and c supernovae are core-collapse supernovae ( $\mathrm{SNe})$ that show no hydrogen, and no hydrogen or helium, respectively, in their optical spectra ${ }^{1}$, and refer to them collectively as 'Type I b/c'. I will also mention on occasion Type IIb supernovae, which are ones that show hydrogen lines early on that subsequently disappear. Type I b/c as well as IIb SNe are thought to arise from the core collapse of a massive star which has lost most or all of its envelope either due to stellar winds or due to mass-transfer to a binary companion prior to the $\mathrm{SN}$ explosion, and for this reason are sometimes known as 'stripped envelope' $\mathrm{SNe}$ (Clocchiatti et al. 1996). They are of especial interest because they have been associated with gamma-ray bursts $(\mathrm{GRB})^{2}$. For a review of the radio observations of GRBs, see Granot \& van der Horst (2013).

\footnotetext{
${ }^{1}$ Note that the classification may be time-dependent. For example, SN 2008D was first classified as being of Type Ic, but later strong He lines emerged and it was re-classified as being of Type Ib (Modjaz et al. 2009). SN 2011ei, on the other hand, appeared to be of Type IIb early on, but had rapidly fading $\mathrm{H}$ lines, so that if observed on the order of 1 week later it would have been classified as Type Ib (Milisavljevic et al. 2013).

${ }^{2}$ In this paper, I will use the general term GRB, but am in fact only referring to long-duration bursts. GRBs are generally classed as long when they have lengths $>2 \mathrm{~s}$. However, there is recent evidence that some bursts as short as $0.8 \mathrm{~s}$ also originate in the collapse of a massive star (Piran et al. 2013), and thus might occur in conjunction with a Type I b/c SN and therefore be of relevance to the discussion in this paper.
}

In this review, I will discuss in particular the results of very long baseline interferometry (VLBI) radio observations of Type I b/c SNe. The primary reason for VLBI observations is that the unmatched resolution of VLBI provides almost the only way of obtaining spatially resolved information about the sources. VLBI can reach angular resolutions of 0.1 milliarcsec, which corresponds to a distance of $\sim 1$ light-month at $50 \mathrm{Mpc}$. VLBI observations therefore potentially allow us to measure the size, shape, and speed of the expanding fireballs.

Generally in supernovae, the radio emission is synchrotron emission, which arises from the combination of magnetic fields and relativistic particles, both of which are produced by the shocks generated by the SN. A particular advantage of radio observations in this field is that radio emission traces the fastest ejecta, which are difficult or impossible to see optically. The explosive ejection of material in a SN generally produces a forward shock where the ejecta impact upon their surroundings, as well as a reverse shock which is driven back into the ejecta. In the well-studied case of the Type IIb SN 1993J, Bartel et al. (2007) give arguments that the outer bounds of the radio-emission region are closely associated with this forward shock location.

It was generally thought that the most common progenitors of Type I b/c SNe were Wolf-Rayet stars (Van Dyk, Li, \& Filippenko 2003a; Woosley, Heger, \& Weaver 2002). However, recently evidence has emerged that suggests that lower mass He stars make up a substantial fraction, perhaps 
the majority, of Type I b/c progenitors (Eldridge et al. 2013; Smith et al. 2011). The progenitors of both classes of SNe are thought to be similar, although there is evidence suggesting that the Type Ic progenitors may be somewhat more massive than the Type Ib ones (Kuncarayakti et al. 2013). However, given that to date there has been no definitive detection of a Type I b/c progenitor, such evidence must remain somewhat inconclusive [although note that Van Dyk, Li, \& Filippenko (2003b) and Cao et al. (2013) report possible detections of both Type Ib and Ic progenitors].

Type I b/c SNe can exhibit very large ejection velocities, in some cases relativistic. SNe where the optical spectrum shows broad lines implying particularly large velocities ( $20000 \mathrm{~km} \mathrm{~s}^{-1}$ ) are often classed as 'broad lined'. Type II $\mathrm{SNe}$, by contrast, have not generally been seen to have high ejection velocities, with the exception of ones of Type IIb, such as SN 2003bg (Hamuy et al. 2009) and SN 2011dh (Marion et al. 2011), which do show high ejection velocities. Only $5-10 \%$ of all Type Ic SNe have broad lines.

Type I b/c SNe are particularly interesting because they have been associated with long-duration GRBs. So far, all the GRBs that have been reliably associated with a spectroscopically confirmed SN have been associated with one of Type I $\mathrm{b} / \mathrm{c}$. In the cases where the spectrum of the $\mathrm{SN}$ allowed a more precise classification, the SNe were of Type Ic with broad lines. Although many, perhaps all GRBs show a supernovalike 'bump' in their light curves, only in relatively nearby examples can the accompanying $\mathrm{SN}$ be identified more conclusively in the spectrum (Bersier 2012). Note, however, that the great majority of Type I b/c SNe are not accompanied by GRBs. In fact, even of the subset of broad-lined Type Ic SNe, only $\sim 20 \%$ are associated with a GRB event.

The most popular model for long-duration GRBs is the so-called collapsar model, (Woosley 1993; MacFadyen, Woosley, \& Heger 2001), in which a core-collapse supernova produces or triggers a central engine (accreting, rapidly spinning compact object). The central engine drives a highly relativistic jet, while the more spherical SN explosion is powered by neutrinos. An observable GRB is produced when the jet is oriented close to the line of sight, and the strong Doppler boosting causes the observed strong gamma-ray emission. Such events also produce emission at lower frequencies, called the afterglow. In particular, radio afterglows are often produced. The radio emission is produced later in the evolution of a GRB, once the velocities have become subrelativistic and the emission isotropised. Unlike the gammaray emission, therefore, the radio emission is not strongly beamed.

So it seems that both the stripped-envelope nature of the supernova and the presence of particularly high ejection velocities are required, but not sufficient for a $\mathrm{SN}$ to produce a GRB. The tentative picture that emerges is that a small subset of Type I b/c SNe are characterised by relativistic ejection velocities, and thus have broad-lined optical spectra. Some of these produce bright, cosmological ('normal') GRBs, which emit $>10^{49.5}$ erg in gamma rays, whereas more of them pro- duce low-luminosity bursts like GRB 0908425, which emit $<10^{48.5} \mathrm{erg}$ in gamma rays. In the case of the bright GRBs, the ejection is highly collimated (opening angles of $\sim 0.1$ radian), and is probably due to a relatively long-lived (at least tens of seconds) jet that emerges from the collapsing star. This jet occurs in conjunction with a more isotropic SN event, but in the case of an observed GRB where the jet is presumably oriented near our line of sight, the emission produced by the jet is usually brighter than that produced by the supernova.

In a bright GRB, the high-energy emission is thought to be produced by shocks internal to the jet. The emission at longer wavelengths, termed the 'afterglow', and in particular the radio emission, is thought to be produced by the external shocks as the jet interacts with the surrounding medium.

In the low-luminosity GRBs, the ejection is likely less collimated (opening angle $\gtrsim 1$ radian), and the high-energy emission is probably associated with the supernova shock breakout (e.g. Kulkarni et al. 1998; Nakar \& Sari 2012), and the jet, if present, does not emerge from the stellar surface, or is relatively weak compared to the more isotropic supernova shock breakout. Note that probably the majority of GRBs are of the low-luminosity variety, and only a minority of GRBs are 'normal', but the latter are far more easily detected (Liang et al. 2007). Soderberg et al. (2006b) showed that the volumetric rate of low-luminosity GRBs is comparable to that of broad-lined Type I b/c SNe.

At present, it is not well understood why these high ejection velocities occur in some $\mathrm{SNe}$, and what causes the collimation. Clearly information as to the size, shape, and speed of the expanding ejecta, such as can be obtained with VLBI, can provide important observational constraints in attempting to unravel these mysteries.

However, SNe that are sufficiently bright to be observable with VLBI occur only rarely. Only a fraction of SNe are ever detected in the radio, and of those, only for ones that are relatively nearby is VLBI imaging useful. For example, after $90 \mathrm{~d}$, a SN expanding with a normal non-relativistic speed of $20000 \mathrm{~km} \mathrm{~s}^{-1}$ could be resolved with $22-\mathrm{GHz}$ VLBI (resolution $\sim 0.2$ mas with a global array) out to a distance of $\sim 5 \mathrm{Mpc}$, while one expanding with an apparent speed of $c$ could be resolved out to $\sim 80 \mathrm{Mpc}$. Given a model of the morphology of the radio emission, geometric models can be fit directly to data in the visibility (Fourier-transform) plane, and angular sizes determined with accuracies which depend on the signal-to-noise, but can be up to $20 \times$ higher than the nominal imaging resolution. Such angular size estimates, however, are dependent on the geometrical model assumed for the emission, which is not well known, in particular for relativistic events. This model uncertainty will usually dominate the uncertainty in the size estimate. Nonetheless, in most cases, angular size estimates can be made with a fractional accuracy of better than $50 \%$, leading to estimates of the apparent expansion velocity of similar accuracy provided the distance and explosion date are well constrained (see Bartel et al. 2002; Bietenholz, Soderberg, \& Bartel 2009, for more detailed discussions of this process). 
Table 1 Type I b/c supernovae observed with VLBI.

\begin{tabular}{lllccl}
\hline \hline Supernova & Type & Galaxy & $\begin{array}{c}\text { Distance } \\
(\mathrm{Mpc})\end{array}$ & $\begin{array}{l}L^{a} \text { peak,8.4 GHz } \\
\left(\times 10^{27} \mathrm{erg} \mathrm{s}^{-1} \mathrm{~Hz}^{-1}\right)\end{array}$ & References \\
\hline SN 1994i & Ic & M51 & 9 & 2 & Bietenholz \& Bartel (unpublished) \\
SN 2001em & I b/c & NGC 7142 & 80 & 12 & $\begin{array}{c}\text { Bietenholz \& Bartel (2005, 2007), Paragi et al. (2005), } \\
\text { Schinzel et al. (2009) }\end{array}$ \\
SN 2003L & I c & NGC 3506 & 92 & 30 & Soderberg et al. (2005) \\
SN 2003gk & I b/c & NGC 7460 & 45 & $<0.5$ & Bietenholz et al. (2013) \\
SN 2007gr & I b/c & NGC 1058 & 10 & 0.1 & Paragi et al. (2007), Soderberg et al. (2010a) \\
SN 2007uy & I b & NGC 2770 & 27 & 1.1 & van der Horst et al. (2011) \\
SN 2008D & I b/c BL ${ }^{b}$ & NGC 2770 & 27 & 2.5 & Bietenholz et al. (2009), van der Horst et al. (2011) \\
SN 2009bb & I b/c BL B ${ }^{b}$ & NGC 3278 & 40 & 50 & Bietenholz et al. (2010b) \\
\hline \hline
\end{tabular}

${ }^{a}$ The peak spectral luminosity of the supernova at $8.4 \mathrm{GHz}$.

$b$ 'BL' indicates a supernova classified as broad-lined due to the presence of broad absorption lines in the early optical spectrum.

In the case of relativistic ejecta, it is possible that the apparent expansion speed becomes superluminal. Indeed, in the case of GRB 030329, associated with SN 2003dh, VLBI observations showing that the radio-emitting region was expanding with an apparent speed of 3-5c during the first few months after the GRB (Taylor et al. 2004, 2005; Pihlström et al. 2007) constitute the most compelling evidence for relativistic expansion in GRB events.

However, given the small fraction of SNe that are sufficiently radio bright that such imaging is possible and near enough that it is useful, the number that has so far been observed is small. Furthermore, since the radio emission from Type I b/c SNe usually fades over a period of months, ${ }^{3}$ the period over which any one SN can be observed will be limited. In this review, I will summarise the constraints that have been so far obtained through such observations.

Note that broadband radio observations of SNe which cannot be resolved by VLBI, provide a second, important albeit less direct constraint on the size of the forward shock region. The broadband spectral energy distributions of $\mathrm{SNe}$ typically are synchrotron self-absorbed below some turnover frequency, which is usually in the range of radio observation (0.1-100 GHz). For a given radio luminosity, the frequency at which synchrotron self-absorption becomes important depends only on the size of the emitting region, and therefore the size can be estimated if the spectral peak can be identified (provided the distance is known). This method is typically useful for measuring the size of the radio-emission region early on in the evolution of the $\mathrm{SN}$ (see, e.g. Chevalier \& Fransson 2006). It is, however, dependent on several assumptions, including that of equipartition between relativistic electrons and magnetic field in the post-shock region. In the case of the Type IIb SN 2011dh, an estimate of size and expansion of the forward shock was obtained in this

\footnotetext{
${ }^{3}$ Some Type II SNe remain radio bright for many years, with notable examples being SN 1993J, SN 1986J, and SN 1979C (see, e.g. Bartel 2009), but Type I b/c SNe usually fade quickly.
}

fashion, with the estimates subsequently confirmed by VLBI measurements (Bietenholz et al. 2012).

Table 1 gives an overview of the Type I b/c SNe which have been observed with VLBI to date. VLBI observations were also obtained of two further $\mathrm{SNe}$, which were discovered in the radio, and are thus of uncertain type: SN 2008iz in M82, which was likely of Type II, but could have been of Type I b/c also (Brunthaler et al. 2010), and the unusual SN 1986J (see e.g. Bietenholz, Bartel, \& Rupen 2010a), which is generally classed as Type IIn, but for which a possible Type Ib classification has also been suggested (Leibundgut et al. 1991). As can be seen, only a small number of $\mathrm{SNe}$ have been observed with VLBI, and in the remainder of this paper, I will discuss most of these relatively rare events in more detail.

\section{SN 2001em AND OTHER 'OFF AXIS' AFTERGLOWS}

A consequence of the idea that GRBs are produced by highly collimated jets oriented near the line of sight is that for each observed GRB, there should be numerous similar events but with the jets not oriented near the line of sight. These events, which I will call 'off-axis' events, and which are also known as orphan afterglows, would not produce observable gammarays. However, since the radio emission mostly arises once the source becomes mildly relativistic, so that its radio emission is more isotropic, the off-axis events should not be significantly more difficult to detect than on-axis ones in the radio. Radio wavelengths were shown to be good for detecting such off-axis events (e.g. Paczyński 2001; Granot \& Loeb 2003), and in a constant density medium, an offaxis jet is expected to peak between 1 and $3 \mathrm{yr}$ after the event (van Eerten, Zhang, \& MacFadyen 2010; Granot \& Loeb 2003). Note, however, that more recent results show that for a wind-stratified medium (with density $\propto r^{-2}$ ), and using a reasonable range for parameters such as the energy of the explosion, the circumstellar density and the efficiency of magnetic field generation and particle acceleration at the shock, a wide variety of lightcurves can be produced, many of 
which are much fainter than the canonical models, and some of which overlap with the expected radio lightcurves of nonrelativistic SNe (Bietenholz et al., 2013). It is therefore likely that only a small fraction of such off-axis relativistic jets will be observably bright in the radio.

Nonetheless, it was suggested that SN $2001 \mathrm{em}$, at a distance of $\sim 80 \mathrm{Mpc}$, and spectroscopically classified as a Type I b/c supernova, might possibly harbour such an off-axis GRB jet on the basis of its unusually high X-ray and radio luminosities, with the latter reaching a maximum only $\sim 1000$ d after the explosion (Granot \& Ramirez-Ruiz 2004, see also Paczyński 2001). Several groups obtained VLBI observations of SN 2001em (Stockdale et al. 2005; Bietenholz \& Bartel 2005; Paragi et al. 2005). The most constraining of these results was that of Bietenholz \& Bartel (2005), obtained at $t \sim 3.2 \mathrm{yr}$, where here and hereafter I will use $t$ to indicate the time interval since shock breakout. The VLBI observations were not consistent with the relativistic expansion expected of a GRB jet, but rather suggested an average expansion velocity of $20000^{+7}{ }_{-12000} \mathrm{~km} \mathrm{~s}^{-1}$, typical of what is found in ordinary non-relativistic SNe.

Subsequent VLBI observations (Bietenholz \& Bartel 2007; Schinzel et al. 2009) have further confirmed the nonrelativistic expansion, with the most recent $3 \sigma$ limit on the expansion speed being $9000 \mathrm{~km} \mathrm{~s}^{-1}$, and have also placed non-relativistic limits on the proper motion (Schinzel et al. 2009). Furthermore, broad $\mathrm{H} \alpha$ lines have appeared in $\mathrm{SN}$ 2001em's optical spectrum, prompting a re-classification to Type IIn (Soderberg, Gal-Yam, \& Kulkarni 2004). An alternate model for SN 2001em, not involving a GRB event or any relativistic ejection, was proposed by Chugai \& Chevalier (2006), which involves the interaction of normal, nonrelativistic, SN ejecta with a massive and dense circumstellar shell, produced by mass loss of the progenitor, and which seems to be able to account for the observational data.

So far, no off-axis events have been detected in blind surveys (e.g., Levinson et al. 2002; Gal-Yam et al. 2006). Note, however, that blind surveys of a sensitivity sufficient to ensure detection of off-axis events at the predicted brightness levels are still prohibitively expensive in observing time.

However, since GRBs arise in conjunction with supernovae, of which many are detected optically, the strategy of looking for off-axis GRB events accompanying known Type $\mathrm{I} \mathrm{b} / \mathrm{c}$ SNe suggested itself. So far, two different searches ${ }^{4}$ for late-time radio emission from Type I b/c SN did not yield any unambiguous detections: Soderberg et al. (2006a) carried out a search for late-time radio emission from Type I b/c $\mathrm{SNe}$, observed $68 \mathrm{SNe}$ at late times with the VLA. A further set of radio observations of 59 Type I b/c SNe was carried out by Bietenholz et al. (2013), who also did not detect any late-time radio emission due to off-axis GRB jets, and concluded that fewer than $<2 \%$ of all Type I b/c SNe, and $<30 \%$

\footnotetext{
${ }^{4}$ Note that Berger et al. (2003) also searched for radio emission from Type $\mathrm{I} \mathrm{b} / \mathrm{c} \mathrm{SNe}$, however, only a few of their observations were at late times $t>1$ $\mathrm{yr}$, and in none of those cases was radio emission detected.
}

of the broad-lined ones, are associated with jet comparable to those seen in bright GRB afterglows. However, they also showed that for more realistic parameters for the circumstellar density as well as the efficiency of particle acceleration and field generation, the radio emission from off-axis jets could be several orders of magnitude lower than previously predicted, and that therefore only a small minority of off-axis jets would likely produce observable radio emission, and that consequently the non-detection of such radio emission so far is readily explained even if a large fraction of SNe Type I b/c do harbour relativistic jets with energies comparable to that of GRB jets.

In Bietenholz et al.'s survey for late-time radio emission, only a single supernova, SN 2003gk, at $\sim 45 \mathrm{Mpc}$, was reliably detected, with an $8.4-\mathrm{GHz}$ spectral luminosity of $\sim 6 \times 10^{27} \mathrm{erg} \mathrm{s}^{-1}$ at $t \simeq 6$ yr. However, VLBI follow-up observations showed that SN 2003gk's average (over $t \simeq 8 \mathrm{yr}$ ) expansion speed was $\sim 10000 \mathrm{~km} \mathrm{~s}^{-1}$, ruling out a relativistic jet, and suggesting that also in this case, interaction with the dense shell in the circumstellar medium was powering the radio emission (Bietenholz et al. 2013).

A further supernova, SN 2007bg, was also suggested to possibly harbour an off-axis jet on the basis of its radio emission (Prieto, Watson, \& Stanek 2009). No VLBI observations of it were undertaken due to its relatively large distance of $152 \mathrm{Mpc}$, however, also in this case, the radio (and X-ray) emission is due to interaction of non-relativistic ejecta with dense CSM resulting from episodic mass-loss from the progenitor rather than to any relativistic ejection (Salas et al. 2013, see also Soderberg 2009).

\section{SN 2008D: JET OR SHOCK BREAKOUT?}

SN 2008D was first discovered as the X-ray flash (XRF) 080109 with the accompanying supernova being discovered soon after in the optical (Soderberg et al. 2008). No gammaray emission was seen even though the source was in the field-of-view of the Burst Alert Telescope. It is at a distance of $\sim 28 \mathrm{Mpc}$, and was, as mentioned above, originally classified as of Type Ic with broad lines, but subsequently re-classified as Type Ib when narrow He lines appeared (e.g. Modjaz et al. 2009).

The origin of the X-ray flash is the subject of some debate. Some authors suggested that it is of supernova-shock origin (Soderberg et al. 2008; Chevalier \& Fransson 2008), while others suggested that it was caused by a mildly relativistic jet, which penetrated the envelope of the progenitor (Mazzali et al. 2008; Li 2008; Xu, Zou, \& Fan 2008).

Radio emission was detected from SN 2008 shortly after shock breakout. Several sets of VLBI observations were obtained. VLBI Observations were obtained with various arrays including telescopes of the NRAO Very Long Baseline Array and the European VLBI Network (EVN) as well as Arecibo, at frequencies of $22,8.4$, and $5.0 \mathrm{GHz}$, and at epochs $t=28$, 30, 69, and $133 \mathrm{~d}$ (Bietenholz et al. 2009; van der Horst et al. 2011). Although the source was not definitively resolved at 

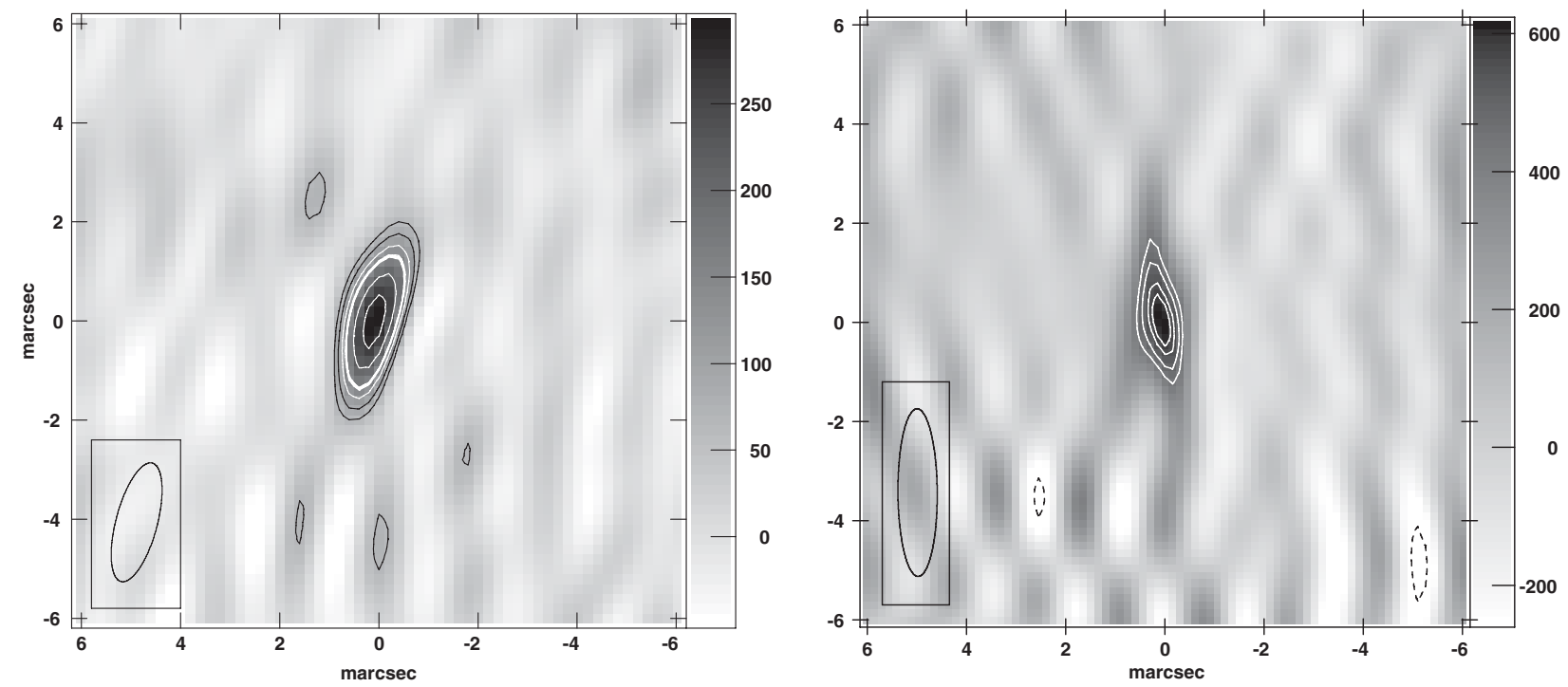

Figure 1. VLBI images of SN 2008D and SN 2009bb. For both images, the lowest contour is drawn at $3 \times$ the image rms background brightness, the greyscale is labelled in $\mu \mathrm{Jy}_{\text {beam }}{ }^{-1}$, the full width at half maximum of the convolving beam is indicated at lower left and north is up and East is to the left. Left: an image of SN 2008D at $5 \mathrm{GHz}$ on 2008 May 21. The contours are drawn at $-21,21,30,40, \mathbf{5 0}, 70$, and $90 \%$ of the peak brightness of $296 \mu \mathrm{Jy}$ beam $^{-1}$, with the $50 \%$ contour being emphasised (from Bietenholz et al. 2009). Right: an image of SN $2009 \mathrm{bb}$ at $8.4 \mathrm{GHz}$ on 2009 June 11 . The contours are at $-57,57,70,80,90$, and $95 \%$ of the peak brightness of $613 \mu \mathrm{Jy} \mathrm{beam}^{-1}$ (see Bietenholz et al. 2010b, for details). I do not consider the extensions to the south of the supernova (visible in the greyscale, but below the $3 \sigma$ contour) to be real.

any of these epochs or frequencies, $3 \sigma$ upper limits on the apparent expansion velocity, $\beta_{\text {app }}$, of $0.71 c$ could be set ${ }^{5}$ from the observations at $t=133 \mathrm{~d}$ (Bietenholz et al. 2009). I show a VLBI image of SN 2008D in Figure 1 (left). van der Horst et al. (2011) obtained similar limits on the $\beta_{\text {app }}$. These limits on $\beta_{\text {app }}$ ruled out a long-lived, highly relativistic outflow, but are still compatible with a rapidly decelerating, mildly relativistic jet. Recent simulations by Bersten et al. (2013) suggest that SN 2008D's bolometric light curve cannot easily be reproduced without the presence of $\sim 0.01 \mathrm{M}_{\odot}$ of ${ }^{56} \mathrm{Ni}$-rich material in the outer layers of the ejecta, which they attribute to the action of such a jet.

\section{SN 2009bb: RELATIVISTIC EJECTA?}

The nearby SN for which there was the best evidence of relativistic expansion was SN 2009bb, detected by the Chilean Automatic SN Search Program (CHASE, Pignata et al. 2009), at a distance of $\sim 40 \mathrm{Mpc}$ in the nearby spiral galaxy NGC 3278. The shock breakout date was unusually well constrained to be March 19 \pm 1 UT. SN 2009bb showed broad lines, implying a photospheric velocity of $\sim 25000 \mathrm{~km} \mathrm{~s}^{-1}$, had a moderate X-ray luminosity, and was quickly found to be radio bright (Soderberg et al. 2010b). Its peak 8.4-GHz spectral luminosity was $\sim 5 \times 10^{28} \mathrm{erg} \mathrm{s}^{-1} \mathrm{~Hz}^{-1}$, larger than that observed for any other SN I b/c at a similar time after shock breakout although only slightly higher than that of SN 1998bw.

\footnotetext{
${ }^{5}$ The quoted limit was for an assumed circular morphology, for an elongated morphology, the $3 \sigma$ upper limit on $\beta_{\text {app }}$ was $2.6 c$ (Bietenholz et al. 2009).
}

Angular sizes derived from fitting a synchrotron selfabsorption model to the broadband radio SED suggested a mean shock velocities of $0.85 \pm 0.02 c$, assuming equipartition of energy between electrons and magnetic fields (Soderberg et al. 2010b).

VLBI observations were undertaken at $t=85 \mathrm{~d}$. Unfortunately, the declination of SN $2009 \mathrm{bb}$ was $-40^{\circ}$, and the paucity of VLBI telescopes in the Southern hemisphere generally renders VLBI observations difficult, and furthermore the South African telescope at Hartebeesthoek, which provides the long baselines crucial for high resolution, was out of commission due to a bearing failure. The observations were obtained at a frequency of $8.4 \mathrm{GHz}$, when the total flux density of the SN at that frequency was $2.5 \mathrm{mJy}$, and I show the VLBI image in Figure 1 (right). Only an upper limit on the angular size could be determined, which was 0.64 mas ( $3 \sigma$; corresponding to a shock velocity of $<1.74 c$; see Bietenholz et al. 2010b for details). The VLBI observations are therefore consistent with, but do not require, moderately relativistic expansion for SN 2009bb.

Chakraborti \& Ray (2011) interpreted SN 2009bb as having a baryon-loaded relativistic blast wave, launched by a central-engine-driven explosion, and predicted that it would be resolvable by VLBI by $t \simeq 200 \mathrm{~d}$. However, by that time, the flux density was already too low ( $\lesssim 1 \mathrm{mJy}$ at $8.4 \mathrm{GHz})$ to warrant further VLBI observations.

In the case of another nearby Type Ic SN, SN 2007gr, 5GHz VLBI observations at $t \simeq 25$ and $85 \mathrm{~d}$ (Paragi et al. 2010) were first interpreted to suggest relativistic expansion. Relativistic expansion was unexpected since the radio luminosity of SN $2007 \mathrm{gr}$ was low, with the $8.4-\mathrm{GHz}$ spectral luminosity 
peaking at $\sim 10^{26} \mathrm{erg} \mathrm{s}^{-1} \mathrm{~Hz}^{-1}$, almost 1000 times lower than that of SN 1998bw. A large angular size and therefore large expansion velocity was suggested because the VLBI observations recovered only a fraction of the total flux density measured at WSRT, suggesting that a significant fraction of the flux density was on scales larger than those measured by VLBI, and therefore setting a lower limit on the size of the source. However, a re-examination by Soderberg et al. (2010a) showed that prolonged relativistic expansion was not likely in this case. In particular, the difference between the total flux density as measured with the Westerbork Synthesis Radio Telescope and that observed even at the shortest VLBI baselines, if interpreted as being due to a resolved source, suggested a source much larger than expected even for credible relativistic expansion. Soderberg et al. (2010a) suggested a more plausible explanation of a somewhat overestimated WSRT total flux density and moderate coherence loss in the VLBI observations. Maeda (2013) also find that the radio light curves of SN 2007gr suggest non-relativistic expansion. Nonetheless, a relativistic ejection that is rapidly ( $<1$ week) decelerated is still compatible with, although by no means required by, the VLBI measurements.

A further supernova, SN 2003L, (92 Mpc), although not showing any broad absorption lines, had a very high radio luminosity, comparable to that of SN 1998bw, which suggested that there might be relativistic ejecta present. It was observed with the NRAO VLBA at $t=65 \mathrm{~d}$ and the results suggested an expansion velocity of only $0.2 c$ (Soderberg et al. 2005).

\section{CONCLUSIONS}

Type I b/c SNe are particularly interesting because they can have relativistic ejecta and because they have been associated with GRBs. However, the nature of the relativistic ejection and the relationship between Type I b/c SNe and GRBs are far from clear. Among the many open questions are: What causes some $\mathrm{SNe}$ to have a relativistic shock breakout? What causes some $\mathrm{SNe}$ to have a comparatively long-lived relativistic jet, which can produce a GRB? Where are the offaxis GRB jets? What is the relationship between GRBs and less extreme supernova? VLBI observations are virtually the only way to obtain spatially resolved information about the explosions, and thus directly constrain the geometry and expansion speeds of the ejection. Unfortunately, $\mathrm{SNe}$ that are close and radio bright enough to be accessible to VLBI are rare, to date only eight Type I b/c SNe have been observed with VLBI (see Table 1).

In none of this small sample observed so far have the VLBI observations produced conclusive evidence of relativistic expansion, or allowed the shape of the emission region to be determined. The VLBI observations have, however, ruled out long-lived relativistic jets such as are thought to produce GRBs in several cases (SN 2001em, SN 2003gk). The case of short-lived, more isotropic relativistic ejection is more difficult. Since the events are short-lived, only in the case of very nearby events does the fireball expand to a size resolvable by VLBI before it becomes too faint to observe. The evidence in these cases is consequently less conclusive, with the VLBI observations obtained so far being compatible with, but not requiring, the mildly relativistic ejecta suggested by observations at other wavelengths in the cases of SN 2008D and SN 2009bb.

Given the importance of direct observational determination of the size, speed, and shape of the expanding shock front, VLBI observations of any $\mathrm{SNe}$ that are sufficiently near and radio bright should be undertaken. With the increasing sensitivity due to both increasing bandwidth, such as the $2 \mathrm{Gbit} \mathrm{s}^{-1}$ recording $\mathrm{GHz}$ bandwidth now available at the NRAO VLBA ${ }^{6}$ (to be upgraded to $4 \mathrm{Gbit} \mathrm{s}^{-1}$ in future), and the incorporation of new and refurbished telescopes into global VLBI networks (see van Langevelde 2013; Gaylard 2013), individual SNe can be observed for longer times, and fainter ones are becoming accessible to VLBI observations. Although the resolution at any particular frequency is limited by the length of the baselines and therefore limited by the Earth's diameter for ground-based telescopes, higher sensitivity also allows observations at higher frequencies with a concomitant increase in angular resolution. Higher resolution could also be obtained by using space-based VLBI antennas such as RadioAstron (Kardashev et al. 2013), however, due to the limited sensitivity of RadioAstron only exceptionally radio-bright $\mathrm{SNe}$ could be studied in this manner. The development of e-VLBI (see, e.g. van Langevelde 2009) offers quick response times, which could be crucial for resolving relatively nearby events early on in their evolution, with the first e-VLBI observations of a SN being those of the Type I b/c SN 2001em of Paragi et al. (2005). The availability of a VLBI array, which operates full time, such as the NRAO VLBA, rather than only in sessions during part of the year, is also of importance due to the often short-lived nature of these events. It will be important also to follow up $\mathrm{SNe}$ not detected in the optical, since the examples of SN 2008iz $(\sim 4 \mathrm{Mpc})$ and SN 1986J ( $\sim 10 \mathrm{Mpc})$ show that even relatively nearby $\mathrm{SNe}$ can go undetected in optical observations.

\section{ACKNOWLEDGEMENTS}

The research at York University was supported by the National Sciences and Engineering Research Council of Canada. I have made use of NASA's Astrophysics Data System Bibliographic Services. I thank N. Bartel, J. Granot, Z. Paragi and A. Gal-Yam for useful comments on the manuscript.

\section{REFERENCES}

Bartel, N. 2009, in Astronomical Society of the Pacific Conference Series, Vol. 402, Approaching Micro-Arcsecond Resolution with VSOP-2: Astrophysics and Technologies, ed. Y. Hagiwara, E. Fomalont, M. Tsuboi, \& M. Yasuhiro,

\footnotetext{
${ }^{6}$ https://science.nrao.edu/facilities/vlba/docs/manuals/oss2013a
} 
Astronomical Society of the Pacific, Sagamihara, Kanagawa, Japan, 243

Bartel, N., Bietenholz, M. F., Rupen, M. P., \& Dwarkadas, V. V. 2007, ApJ, 668, 924

Bartel, N., et al. 2002, ApJ, 581, 404

Berger, E., Kulkarni, S. R., Frail, D. A., \& Soderberg, A. M. 2003, ApJ, 599, 408

Bersier, D. 2012, ArXiv e-prints, arXiv:1206.6979

Bersten, M. C., Tanaka, M., Tominaga, N., Benvenuto, O. G., \& Nomoto, K. 2013, ApJ, 767, 143

Bietenholz, M. F., De Colle, F., Granot, J., Bartel, N., \& Soderberg, A. M. 2013, ArXiv e-prints, arXiv: 1310.7171

Bietenholz, M. F., \& Bartel, N. 2005, ApJL, 625, L99

Bietenholz, M. F., \& Bartel, N. 2007, ApJL, 665, L47

Bietenholz, M. F., Bartel, N., \& Rupen, M. P. 2010a, ApJ, 712, 1057

Bietenholz, M. F., et al. 2012, ApJ, 751, 125

Bietenholz, M. F., Soderberg, A. M., \& Bartel, N. 2009, ApJL, 694, L6

Bietenholz, M. F., et al. 2010b, ApJ, 725, 4

Brunthaler, A., et al. 2010, A\&A, 516, A27

Cao, Y., et al. 2013, ApJL, 775, L7

Chakraborti, S., \& Ray, A. 2011, ApJ, 729, 57

Chevalier, R. A., \& Fransson, C. 2006, ApJ, 651, 381

Chevalier, R. A., \& Fransson, C. 2008, ApJL, 683, L135

Chugai, N. N., \& Chevalier, R. A. 2006, ApJ, 641, 1051

Clocchiatti, A., Wheeler, J. C., Benetti, S., \& Frueh, M. 1996, ApJ, 459,547

Eldridge, J. J., Fraser, M., Smartt, S. J., Maund, J. R., \& Crockett, R. M. 2013, MNRAS, 436, 774

Gal-Yam, A., Ofek, E. O., Poznanski, D., et al. 2006, ApJ, 639, 331

Gaylard, M. J. 2013, Materials Science and Engineering Conference Series, 44, 012020

Granot, J., \& Loeb, A. 2003, ApJL, 593, L81

Granot, J., \& Ramirez-Ruiz, E. 2004, ApJL, 609, L9

Granot \& van der Horst 2013, PASA, 31

Hamuy, M., et al. 2009, ApJ, 703, 1612

Kardashev, N. S., et al. 2013, ARep, 57, 153

Kulkarni, S. R., et al. 1998, Nat., 395, 663

Kuncarayakti, H., et al. 2013, AJ, 146, 31

Leibundgut, B., Kirshner, R. P., Pinto, P. A., Rupen, M. P., Smith, R. C., Gunn, J. E., \& Schneider, D. P. 1991, ApJ, 372, 531

Levinson, A., Ofek, E. O., Waxman, E., \& Gal-Yam, A. 2002, ApJ, 576,923

Li, L.-X. 2008, MNRAS, 388, 603

Liang, E., Zhang, B., Virgili, F., \& Dai, Z. G. 2007, ApJ, 662, 1111

MacFadyen, A. I., Woosley, S. E., \& Heger, A. 2001, ApJ, 550, 410

Maeda, K. 2013, ApJ, 762, 14

Marion, G. H., et al. 2011, ATel, 3435, 1

Mazzali, P. A., et al. 2008, Sci., 321, 1185

Milisavljevic, D., et al. 2013, ApJ, 767, 71

Modjaz, M., et al. 2009, ApJ, 702, 226

Murphy, T., et al. 2013, PASA, 30, 6
Nakar, E., \& Sari, R. 2012, ApJ, 747, 88

Paczyński, B. 2001, AcA, 51, 1

Paragi, Z., Garrett, M. A., Paczyński, B., Kouveliotou, C., Szomoru, A., Reynolds, C., Parsley, S. M., \& Ghosh, T 2005, MmSAI, 76, 570

Paragi, Z., Kouveliotou, C. Garrett, M. A., Ramirez-Ruiz, E., van Langevelde, H. J., Szomoru, A., \& Argo, M. 2007, ATel, 1215,1

Paragi, Z., et al. 2010, Nat., 463, 516

Pignata, G., et al. 2009, CBET, 1731, 1

Pihlström, Y. M., Taylor, G. B., Granot, J., \& Doeleman, S. 2007, ApJ, 664, 411

Piran, T., Bromberg, O., Nakar, E., \& Sari, R. 2013, Royal Society of London Philosophical Transactions Series A, 371, 20273

Prieto, J. L., Watson, L. C., \& Stanek, K. Z. 2009, ATel, 2065, 1

Salas, P., Bauer, F. E., Stockdale, C., \& Prieto, J. L. 2013, MNRAS, 428, 1207

Schinzel, F. K., Taylor, G. B., Stockdale, C. J., Granot, J., \& Ramirez-Ruiz, E. 2009, ApJ, 691, 1380

Smith, N., Li, W., Filippenko, A. V., \& Chornock, R. 2011, MNRAS, 412,1522

Soderberg, A. M. 2009, ATel, 2066, 1

Soderberg, A. M., Brunthaler, A., Nakar, E., Chevalier, R. A., \& Bietenholz, M. F. 2010a, ApJ, 725, 922

Soderberg, A. M., Gal-Yam, A., \& Kulkarni, S. R. 2004, GRB Circular Network, 2586, 1

Soderberg, A. M., Kulkarni, S. R., Berger, E., Chevalier, R. A., Frail, D. A., Fox, D. B. \& Walker, R. C. 2005, ApJ, 621, 908

Soderberg, A. M., Nakar, E., Berger, E., \& Kulkarni, S. R. 2006a, ApJ, 638, 930

Soderberg, A. M., et al. 2006b, Nat., 442, 1014

Soderberg, A. M., et al. 2008, Nat., 453, 469

Soderberg, A. M., et al. 2010b, Nat., 463, 513

Stockdale, C. J., et al. 2005, IAU Circ., 8472, 4

Taylor, G. B., Frail, D. A., Berger, E., \& Kulkarni, S. R. 2004, ApJL, 609, L1

Taylor, G. B., Momjian, E., Pihlström, Y., Ghosh, T., \& Salter, C. 2005, ApJ, 622, 986

van der Horst, A. J., et al. 2011, ApJ, 726, 99

Van Dyk, S. D., Li, W., \& Filippenko, A. V. 2003a, PASP, 115, 1

Van Dyk, S. D., Li, W., \& Filippenko, A. V. 2003b, PASP, 115, 1

van Eerten, H., Zhang, W., \& MacFadyen, A. 2010, ApJ, 722, 235

van Langevelde, H. J. 2009, in Proceedings of the 8th International e-VLBI Workshop. 2009 June 22-26. Madrid, Spain. Published online at: http://pos.sissa.it/cgi-bin/reader/conf.cgi?confid=82., p. 30

van Langevelde, H. J. 2013, ArXiv e-prints, arXiv:1301.1060

Woosley, S. E. 1993, ApJ, 405, 273

Woosley, S. E., Heger, A., \& Weaver, T. A. 2002, RvMP, 74, 1015

Xu, D., Zou, Y.-C., \& Fan, Y. Z. 2008, ArXiv e-prints, arXiv: 0801.4325 